\title{
Quarantine: use with care
}

Steve Kisely

This editorial discusses the psychological effects of isolation and quarantine in terms of both the SARS-CoV-2 (COVID-19) pandemic and previous epidemics in the past 20 years. Although much of the literature is based on healthcare settings, there is emerging evidence from home or hotel quarantine, particularly concerning international travellers. Regardless of setting, depression, anxiety, anger and stress-related disorders are especially common but can vary according to demographic features and the characteristics of quarantine. Psychological effects may be minimised by clear and consistent advice from authorities, adequate supplies to meet basic needs, and minimising both the duration and the associated financial burden. There should also be adequate protection from possible infection and thus the resulting fear of contracting COVID-19 while in quarantine.

\section{Keywords}

Stigma and discrimination; depressive disorders; post-traumatic stress disorder; anxiety disorders; ethics.

\section{Copyright and usage}

(C) The Author(s), 2021. Published by Cambridge University Press on behalf of the Royal College of Psychiatrists. This is an Open Access article, distributed under the terms of the creative Commons Attribution-NonCommercial-ShareAlike licence (https://creativecommons.org/licenses/by-nc-sa/4.0/), which permits non-commercial re-use, distribution, and reproduction in any medium, provided the same Creative Commons licence is included and the original work is properly cited. The written permission of Cambridge University Press must be obtained for commercial re-use.
Steve Kisely, Princess Alexandra Hospital, Woolloongabba, Australia; School of Medicine, University of Queensland, Australia; and Departments of Psychiatry, Community Health and Epidemiology, Dalhousie University, Canada

Quarantine has been an important public health measure during viral epidemics in the past 20 years, including outbreaks of severe acute respiratory syndrome (SARS) in 2003, H1N1 influenza in 2009, Middle East respiratory syndrome (MERS) in 2012, Ebola in 2014 and SARS-CoV-2 (COVID-19) in 2019.

Last's Dictionary of Epidemiology defines quarantine as the separation and restriction of movement of people who have potentially been exposed to a contagious disease to see if they become unwell. ${ }^{1}$ The duration is determined by the longest usual incubation period, and it can be absolute or selective. An example of the latter would be close supervision of contacts without restricting their movements so as to allow early recognition of symptoms.

This is different from isolation, which is the separation from others of people who actually have been diagnosed with an infectious disease. ${ }^{1}$ In practice, and during the current COVID-19 pandemic, there is a great deal of overlap. Single-person quarantine or isolation is distinct from restrictions that are placed on a whole population. Such lockdowns are outside the scope of this paper. ${ }^{2,3}$

By definition, quarantine is stressful given that it entails boredom, confinement, fear of infection, and isolation from family and friends. ${ }^{4}$ In a rapid review of healthcare workers who cared for affected patients during the current pandemic, as well as in previous viral outbreaks, the fear and actual experience of quarantine were among the strongest predictors of distress. ${ }^{5}$

There have also been several recent rapid, systematic or umbrella reviews of the psychological effects of quarantine both during the current pandemic and in earlier epidemics that included additional population groups. ${ }^{4,6,7}$ Although these covered a wide range of infectious agents such as Ebola, H1N1, MERS, SARS, COVID-19, and methicillin-resistant Staphylococcus aureus, findings seem consistent across settings. Importantly, recent reviews have generally included papers with non-quarantined controls, thus offering stronger evidence than uncontrolled designs. Most of the studies included in the reviews used quantitative assessments of psychological outcomes such as standardised psychiatric instruments. There were high levels (up to $70 \%$ ) of depression, anxiety, anger, and stress-related disorders or post-traumatic stress disorder in both cross-sectional and cohort designs. ${ }^{4,7}$ In one meta-analysis, people in quarantine were nearly three times more likely to be depressed or stressed, and twice as likely to be anxious than non-quarantined controls. ${ }^{6}$ Other adverse effects included boredom, problem drinking and loneliness. Following release from quarantine, social avoidance and anxiety were common.,

Demographic factors associated with greater psychological distress include younger age, being female, lower educational or income levels, financial loss, a past psychiatric history and poor perceived general health. ${ }^{4,6}$ Depending on the setting and infectious agent, stigma is a major issue both during and after quarantine., Protective factors include a positive attitude towards the need for quarantine and continued contact with family through electronic means. ${ }^{4,5}$ Conditions of isolation or quarantine are also important. Worse psychological outcomes are associated with longer duration, fear of infection, inadequate basic supplies, and insufficient or unclear information from relevant authorities. ${ }^{4,5}$

A lot of information on single-person quarantine comes from healthcare settings. The paper by Regehr and colleagues on the psychological consequences of quarantine for travellers arriving in Canada is thus a welcome addition to the literature. ${ }^{8}$ As in other countries, all arriving international passengers apart from essential workers were subject to a mandatory 14-day quarantine, which could be in either a private residence or a rented facility. They were unable to leave their location for any reason except for an emergency. Nearly 11000 international arrivals at Toronto's Pearson Airport were assessed on arrival and on days 7 and 14 using a validated rating tool, the five-item World Health Organization Well-Being Index. They were also asked about attitudes towards COVID-19 and protection behaviours. The proportion of participants with poor mental health rose from $5.1 \%$ on arrival to $26.0 \%$ at 7 days and $27.0 \%$ after 14 days of quarantine. As in previous studies, being younger and being female were associated with poorer psychological outcomes, whereas regarding quarantine as necessary was protective. People who followed public health protection measures also fared better, possibly indicating great confidence in advice from authorities. Although arrivals from Europe were more likely to develop poor mental health, there was no information on any effect on mental health of the reason for travel or whether the traveller was returning home or was on the outward leg of their journey. 
Of course, these findings apply to isolation or quarantine at home or in a rental facility, not hotel quarantine. Hotels have been used to quarantine international arrivals in Australia, New Zealand and Singapore from early 2020, and the practice has subsequently spread to other jurisdictions. Indeed, within Australia, hotel quarantine had been used domestically for inter-state travellers.

It is likely that hotel quarantine would be associated with worse mental health outcomes than quarantine in private dwellings, given the more cramped physical conditions, overcrowding for families placed in one room, the lack of fresh air and monotonous catering. To this is added the not inconsiderable cost of around $\mathrm{A} \$ 3000$ that is charged each adult traveller. Furthermore, the distinction between quarantine and isolation has been blurred, with COVID-19-positive cases being accommodated alongside people who are free of infection. This has led to several examples of cross-infection, particularly with the alpha and delta strains, and therefore the very understandable fear of contracting COVID-19 while in quarantine. Airborne transmission has occurred to the extent that briefly opening doors to the corridor of adjacent hotel rooms at roughly the same time to collect food has led infection passing from one room to the next. ${ }^{9}$ In addition, several community outbreaks of COVID-19 in Australia and New Zealand have been linked to breaches of hotel quarantine. For instance, there were seven breaches in Australia up to 31 January 2021, with one (in Victoria) causing over 800 deaths and six precipitating lockdowns, while in New Zealand there were nine, resulting in three deaths and one lockdown. ${ }^{10}$ There have been several more during 2021. The resulting lockdowns are associated with increased psychological morbidity in the community. For instance, the Victorian lockdown secondary to breaches in hotel quarantine that resulted in 800 deaths was also associated with a $20 \%$ rise in billable psychiatry consultations compared with the same time the previous year. ${ }^{11}$ This was not matched in the rest of Australia. It makes little sense to effectively place quarantine stations for a highly infectious illness in the central business districts of major cities. This means that more people experience the material and psychological consequences of lockdowns arising from breaches than if quarantine had occurred in more remote areas. There has also been an unclear or inconsistent application of quarantine rules in that celebrities can access special arrangements, where they are housed in mansions as long as they pay for security to ensure compliance with quarantine rules. ${ }^{12}$ The resulting perception of inequity may undermine confidence in the necessity of quarantine and thus lead to worse psychological outcomes. ${ }^{8}$

To date, there has been only one study of the psychological effects of hotel quarantine. ${ }^{13}$ This was a study from Sydney, Australia, of emergency department presentations in one of the major local hospitals. Of 2774 people in hotel quarantine, 461 (16.6\%) presented at least once to the emergency department, the most common reason being mental health problems $(n=102)$. The three most frequent psychiatric symptoms were anxiety $(n=43)$, suicidal ideation $(n=24)$ and acute psychosis $(n=11)$. The majority had a psychiatric history. Importantly, mental health presentations had a greater acuity than any of the others, with 86 of the 102 presentations coded as urgent or potentially life-threatening. Given the difficulties in transporting people using full personal protective equipment, this is likely to greatly underrepresent actual psychological morbidity

What can be done to address the adverse psychological effects of quarantine? Good practice would indicate clear and consistent advice from authorities, adequate supplies to meet basic needs, and minimising both the duration and associated financial burden. ${ }^{4}$ There should also be adequate protection from possible infection and thus the fear of contracting COVID-19 while in quarantine. The Australian and New Zealand experience of hotel quarantine falls far short of this ideal. If it is not possible for people to quarantine at home, they should be accommodated in purpose-built facilities with separate standalone units for each traveller or group of travellers, with adequate ventilation and fresh air, and at minimal financial cost to the individual. The only example of a facility in Australia that has these characteristics is Howard Springs, a former worker's camp in the Northern Territory. The success of this approach is illustrated by the fact that there were no quarantine breaches at this facility from 2020 to 2021. In any such setting, there should be screening for prior mental health problems and adequate psychological support. These findings are relevant for any other countries considering quarantine that is not in an individual's home. Although quarantine is necessary in some circumstances, it should be used with care.

Steve Kisely (D)
Correspondence: Steve Kisely. Email: s.kisely@uq.edu.au

First received 9 Aug 2021, final revision 25 Aug 2021, accepted 27 Aug 2021

Funding

None.

Declaration of interest

None.

\section{References}

1 Last JM. A Dictionary of Epidemiology. Oxford University Press, 1988.

2 de Lima CVC, Cândido EL, da Silva JA, Albuquerque LV, de Menezes Soares L, do Nascimento MM, et al. Effects of quarantine on mental health of populations affected by Covid-19. J Affect Disord 2020; 275: 253-4.

3 Yang Q, Wang Y, Tian C, Chen Y, Mao J. The experiences of community-dwelling older adults during the COVID-19 Lockdown in Wuhan: a qualitative study. J AdV Nurs [Epub ahead of print] 14 Jul 2021. Available from: https://doi.org/10.1111/ jan.14978.

4 Brooks SK, Webster RK, Smith LE, Woodland L, Wessely S, Greenberg N, et al. The psychological impact of quarantine and how to reduce it: rapid review of the evidence. Lancet 2020; 395: 912-20.

5 Kisely S, Warren N, McMahon L, Dalais C, Henry I, Siskind D. Occurrence, prevention, and management of the psychological effects of emerging virus outbreaks on healthcare workers: rapid review and meta-analysis. BMJ 2020; 369: m1642.

6 Henssler J, Stock F, van Bohemen J, Walter H, Heinz A, Brandt L. Mental health effects of infection containment strategies: quarantine and isolation-a systematic review and meta-analysis. Eur Arch Psychiatry Clin Neurosci 2021; 271: 223-34.

7 Hossain MM, Sultana A, Purohit N. Mental health outcomes of quarantine and isolation for infection prevention: a systematic umbrella review of the global evidence. Epidemiol Health 2020; 42: e2020038.

8 Regehr C, Goel V, De Prophetis E, Jamil M, Mertz D, Rosella LC, et al. Mental health impacts of quarantine: insights from the coviD-19 international border surveillance study in Toronto, Canada. BJPsych Open 2021; 7(5): e143.

9 Hyde Z, Berger D, Miller A. Australia must act to prevent airborne transmission of SARS-CoV-2. Med J Aust 2021; 215: 7-9.e1.

10 Grout LM, Katar A, Ouakrim DA, Summers JA, Kvalsvig A, Baker MG, et al. Estimating the failure risk of hotel-based quarantine for preventing COVID-19 outbreaks in Australia and New Zealand. medRxiv [Preprint] 2021. Available from: https://doi.org/10.1101/2021.02.17.21251946.

11 Looi JCL, Allison S, Kisely SR, Pring W, Reay RE, Bastiampilla T. Greatly increased Victorian outpatient private psychiatric care during the COVID-19 pandemic: new MBS-telehealth-item and face-to-face psychiatrist office-based services from April-September 2020. Australas Psychiatry 2021; 29(4): 423-9.

12 Mao F. Celebrities in Australia anger stranded citizens over 'double standard'. BBC News. (https://www.bbc.com/news/world-australia-55851074 [cited 02 Aug 2021]).

13 Dinh M, Hutchings O, Bein K, Shaw J, Raut A, Russell SB, et al. Emergency department presentations by residents of Sydney quarantine hotels during the COVID-19 outbreak. Med J Aust 2021; 214: 473-4. 